\title{
ON THE CHROMATIC NUMBER OF SUBSETS OF THE EUCLIDEAN PLANE
}

\author{
M. AXENOVICH, J. CHOI, M. LASTRINA, T. MCKAY, J. SMITH, AND B. STANTON
}

\begin{abstract}
The chromatic number of a subset of the real plane is the smallest number of colors assigned to the elements of that set such that no two points at distance 1 receive the same color. It is known that the chromatic number of the plane is between 4 and 7 . In this note, we determine the bounds on the chromatic number for several classes of subsets of the plane such as extensions of the rational plane, sets in convex position, infinite strips, and parallel lines.
\end{abstract}

\section{INTRODUCTION}

Let $(X, d)$ be a graph with vertex set $X \subseteq \mathbb{R}^{2}$, and $u \sim v$ if and only if the Euclidean distance $\|\cdot\|$ between them is $d$. When $d=1$, this graph is called a unit distance graph. It is well known that $4 \leq \chi\left(\mathbb{R}^{2}, d\right) \leq 7$, for any positive $d$. In the literature, $\chi\left(\mathbb{R}^{2}, 1\right)$ is referred to as the chromatic number of the plane, and the problem of determining its exact value is the famed Hadwiger-Nelson problem. The body of work on this problem is extensive (see for example expository works [16], [4]).

One important result is the following theorem, which allows the problem to be reduced to finite subsets of the plane.

Theorem 1.1 (de Bruijn and Erdős [5]). If $k$ is a positive integer and $G$ is a graph such that any finite subgraph is $k$-colorable, then $\chi(G) \leq k$.

In this paper, we examine special subsets of the plane and investigate the chromatic number of the corresponding graph. In Section 2, we bound $\chi(X, 1)$, where $X$ belongs to a class of subsets of $\mathbb{Q} \times \mathbb{R}$. In Section 3 , we prove that $\chi(X, 1) \leq 4$ if $X$ corresponds to the vertex set of a convex $|X|$-gon. Finally, in Section 4 , we investigate the chromatic number of infinite strips and unions of lines.

\section{On the Chromatic NUmber of SUbSets of $\mathbb{Q} \times \mathbb{R}$}

The fact that $\chi\left(\mathbb{Q}^{2}, 1\right)=\chi\left(\mathbb{Z}^{2}, d\right)=2$ for any natural $d$ was first shown by Woodall [17], and this result has since been reproven independently by many others. A natural next question is determining $\chi(\mathbb{Q} \times \mathbb{R}, 1)$. We know that $\chi(\mathbb{Q} \times \mathbb{R}, 1) \geq 3$, since the triangle with vertices $(0,0),(1,0)$, and $\left(\frac{1}{2}, \frac{\sqrt{3}}{2}\right)$ forms a subgraph of this graph. Is it true that $\chi(\mathbb{Q} \times \mathbb{R}, 1)=4$ ? Is it true that $\chi(\mathbb{Q} \times \mathbb{R}, 1)<\chi\left(\mathbb{R}^{2}, 1\right)$ ? While we cannot answer these questions, we provide some partial results.

Date: April 21, 2010.

2010 Mathematics Subject Classification. Primary 05C12, 05C15, 05C62, 51K99.

Supported in part by NSA grant H98230-09-1-0063 and NSF grant DMS-0901008. 
For the following discussion, let $\mathbb{N}^{*}$ be the set of square-free natural numbers, i.e., the natural numbers that are not divisible by $4,9,16$, etc. For distinct $N_{1}, \ldots, N_{k} \in \mathbb{N}^{*}$, let $\mathbb{Z}\left(N_{1}, N_{2}, \ldots, N_{k}\right)=\left\{a_{1} \sqrt{N_{1}}+\cdots+\right.$ $\left.a_{k} \sqrt{N_{k}}: a_{1}, \ldots, a_{k} \in \mathbb{Z}\right\}$.

Theorem 2.1. $\chi(\mathbb{Q} \times \mathbb{R}, 1)=\max \left\{\chi\left(\mathbb{Z} \times \mathbb{Z}\left(N_{1}, N_{2}, \ldots, N_{k}\right), d\right): N_{1}, \ldots, N_{k} \in \mathbb{N}^{*}, d, k \in \mathbb{N}\right\}$.

Proof. Let $H$ be a finite connected subgraph of $(\mathbb{Q} \times \mathbb{R}, 1)$ with vertex set $\left\{\mathbf{v}_{0}=(0,0), \mathbf{v}_{1}=\left(p_{1} / q_{1}, x_{1}\right), \ldots, \mathbf{v}_{n}=\right.$ $\left.\left(p_{n} / q_{n}, x_{n}\right)\right\} \subseteq \mathbb{Q} \times \mathbb{R}$, where $p_{i}, q_{i} \in \mathbb{Z}$ and $q_{i} \neq 0$ for $i=1, \ldots, n$. Let $d$ be the least common multiple of the $q_{i}$, $1 \leq i \leq n$. Consider the graph $H^{\prime}$ on vertex set $d \mathbf{v}_{1}, \ldots, d \mathbf{v}_{n}$, where two vertices are adjacent if and only if the Euclidean distance between them is $d$. We see that $H$ is isomorphic to $H^{\prime}$, and $H^{\prime}$ is a subgraph of $(\mathbb{Z} \times \mathbb{R}, d)$ that also contains the origin.

We now show that each vertex of $H^{\prime}$ is $(x, y)$, where $x \in \mathbb{Z}$ and $y$ is an integer combination of square roots of positive integers. Let $\mathbf{0}=\mathbf{u}_{\mathbf{0}}, \mathbf{u}_{\mathbf{1}}, \ldots, \mathbf{u}_{\mathbf{m}}$ be a path in $H^{\prime}$ such that $\mathbf{u}_{\mathbf{0}}, \ldots, \mathbf{u}_{\mathbf{m}-\mathbf{1}}$ have such a representation, and consider $\mathbf{u}_{\mathbf{m}}$. We have that $\left\|\mathbf{u}_{\mathbf{m}-\mathbf{1}}-\mathbf{u}_{\mathbf{m}}\right\|=d$. Let $\mathbf{u}_{\mathbf{m}-\mathbf{1}}=\left(b, a_{1} \sqrt{N_{1}}+\cdots+a_{k} \sqrt{N_{k}}\right)$ and $\mathbf{u}_{\mathbf{m}}=\left(b^{\prime}, y\right)$, for $b^{\prime}, b, a_{1}, \ldots, a_{k} \in \mathbb{Z}$, and $y \in \mathbb{R}$. Then

$$
\left(b-b^{\prime}\right)^{2}+\left(a_{1} \sqrt{N_{1}}+\cdots+a_{k} \sqrt{N_{k}}-y\right)^{2}=d^{2}
$$

So, $a_{1} \sqrt{N_{1}}+\cdots+a_{k} \sqrt{N_{k}}-y= \pm \sqrt{d^{2}-\left(b-b^{\prime}\right)^{2}}$. Since $d^{2}-\left(b-b^{\prime}\right)^{2} \in \mathbb{N}$, we have that $y$ is also an integer combination of square roots of positive integers, as desired. To complete the proof, we apply Theorem 1.1.

A lot of work has been done on determining $\chi(\mathbb{Z} \times \mathbb{Z}(N), d)$ for $N \in \mathbb{N}^{*}$ and $d \in \mathbb{N}$. An interesting question is whether $\chi\left(\mathbb{Z} \times \mathbb{Z}\left(N_{1}, N_{2}, \ldots, N_{k}\right), d\right) \leq \max \left\{\chi(\mathbb{Z} \times \mathbb{Z}(N), d): N \in \mathbb{N}^{*}\right\}$, for any $N_{1}, \ldots, N_{k} \in \mathbb{N}^{*}$ and $d \in \mathbb{N}$. Theorem 2.2 summarizes the results of Johnson [11], Fischer [9], Moorehouse [14] and others.

Theorem 2.2. Let $N$ and $d$ be positive integers. Then

$$
\chi(\mathbb{Z} \times \mathbb{Z}(N), d) \leq\left\{\begin{aligned}
& 2, \text { if } N \not \equiv 3(\bmod 4) ; \\
& 3, \text { if } N \neq 2(\bmod 3) ; \\
& 4, \text { if } N \equiv 3(\bmod 8), N \neq 17,59,83(\bmod 84), \text { or } \\
& N \neq 3,5,6(\bmod 7) .
\end{aligned}\right.
$$

Here, we prove a more general statement.

Theorem 2.3. If $d, k \in \mathbb{N}$ and $N_{1}, \ldots, N_{k} \in \mathbb{N}^{*}$, then

$$
\chi\left(\mathbb{Z} \times \mathbb{Z}\left(N_{1}, \ldots, N_{k}\right), d\right) \leq \begin{cases}2, & \text { if } d \not \equiv 0(\bmod 2) \text { or } \text { if } N_{i} \not \equiv 3(\bmod 4) \text { for } 1 \leq i \leq k ; \\ 3, & \text { if } d \neq 0(\bmod 3) \text { or if } N_{i} \not \equiv 2(\bmod 3) \text { for } 1 \leq i \leq k ; \\ 4, & \text { if } d \equiv 2(\bmod 4) \text { or if } N_{i} \not \equiv 7(\bmod 8) \text { for } 1 \leq i \leq k ; \\ 6, & \text { if } d \neq 0(\bmod 12) \quad \text { or if } N_{i} \not \equiv 11(\bmod 12) \text { for } 1 \leq i \leq k .\end{cases}
$$

Proof of Theorem 2.3. The proof is based on several observations concerning number theoretic properties of vertices of the graph under consideration. Let $V=\mathbb{Z} \times \mathbb{Z}\left(N_{1}, \ldots, N_{k}\right)$, where $N_{1}, \ldots, N_{k} \in \mathbb{N}^{*}$. We state several claims and provide their proofs in the Appendix. 
Claim 1. Let $\mathbf{u}, \mathbf{v} \in V$, such that $\|\mathbf{u}-\mathbf{v}\|=d$, an integer. Then there exist $N \in\left\{N_{1}, N_{2}, \ldots, N_{k}\right\}$ and $a, b \in \mathbb{Z}$ such that $\mathbf{u}-\mathbf{v}=(a, b \sqrt{N})$.

Claim 2. Let $\gamma, q, a, b, N \in \mathbb{Z}, d=2^{\gamma} q$, where $\gamma \geq 0, q$ is odd, $N \in \mathbb{N}^{*}$, and $a^{2}+b^{2} N=d^{2}$. Then

(1) $(\gamma=0) \Longrightarrow(a+b N \not \equiv 0(\bmod 2))$;

(2) $(\gamma \geq 1) \Longrightarrow(a \equiv b(\bmod 2))$;

(3) $(\gamma \geq 1$ and $N \not \equiv 3(\bmod 4)) \Longrightarrow\left(a\right.$ and $b$ are multiples of $\left.2^{\gamma}\right)$;

(4) $(\gamma \geq 2$ and $N \not \equiv 7(\bmod 8)) \Longrightarrow\left(a\right.$ and $b$ are multiples of $\left.2^{\gamma-1}\right)$.

Claim 3. Let $\gamma, q, a, b, N \in \mathbb{Z}, d=3^{\gamma} q$, where $\gamma \geq 0, q$ is not a multiple of $3, N \in \mathbb{N}^{*}$, and $a^{2}+b^{2} N=d^{2}$. Then

(1) $(\gamma=0) \Longrightarrow(a+b N \not \equiv 0(\bmod 3))$;

(2) $(\gamma \geq 1) \Longrightarrow\left(a^{2} \equiv b^{2}(\bmod 3)\right)$;

(3) $(\gamma \geq 1$ and $N \not \equiv 2(\bmod 3)) \Longrightarrow\left(a\right.$ and $b$ are multiples of $\left.3^{\gamma}\right)$.

Now we proceed with the proof of Theorem 2.3. Let $G$ be a connected component of $(V, d)$ and assume without loss of generality that it contains the origin. In each of the cases addressed by the theorem, we provide an explicit coloring, $c$, of $G$, and check that it is proper, taking $\mathbf{u}, \mathbf{v} \in V$ such that $\|\mathbf{u}-\mathbf{v}\|=d$ and verifying that $c(\mathbf{u}) \neq c(\mathbf{v})$.

By Claim $1, \mathbf{u}-\mathbf{v}=(a, b \sqrt{N})$, where $a, b \in \mathbb{Z}$ and $N \in\left\{N_{1}, \ldots, N_{k}\right\}$. Moreover, $d^{2}=a^{2}+b^{2} N$.

For each vertex, we introduce the notation:

$$
\mathbf{v}=\left(a_{\mathbf{v}}, \sum_{i=1}^{k} b_{\mathbf{v} i} \sqrt{N_{i}}\right) .
$$

(1) (a) Suppose $d$ is odd. Let $c(\mathbf{v})=a_{\mathbf{v}}+\sum_{i=1}^{k} b_{\mathbf{v} i} N_{i}(\bmod 2)$. Then $d \equiv d^{2}(\bmod 2) \equiv a^{2}+b^{2} N$ $(\bmod 2) \equiv a+b N \equiv|c(\mathbf{v})-c(\mathbf{u})|(\bmod 2)$. Since $d$ is odd, $a+b N$ is odd, thus $c(\mathbf{u}) \neq c(\mathbf{v})$.

(b) Suppose $N_{i} \not \equiv 3(\bmod 4)$ for $i=1, \ldots, k$ and $d=2^{\gamma} q$, where $\gamma \geq 1$ and $q$ is odd. Using Claim 2 (3) and the inclusion of the origin in the vertices of $G$, we see that the coordinates of each vertex in $G$ are divisible by $2^{\gamma}$, so $G$ is isomorphic to a subgraph of $\left(\mathbb{Z} \times \mathbb{Z}\left(N_{1}, \ldots, N_{k}\right), q\right)$, which is bipartite by the previous case.

(2) (a) Suppose $d$ is not a multiple of 3. Let $c(\mathbf{v})=a_{\mathbf{v}}+\sum_{i=1}^{k} b_{\mathbf{v} i} N_{i}(\bmod 3)$. From Claim $3(1), a+b N \not \equiv 0$ $(\bmod 3)$. Thus $c(\mathbf{u}) \neq c(\mathbf{v})$.

(b) Suppose $N_{i} \not \equiv 2(\bmod 3)$ for $i=1, \ldots, k$ and $d=3^{\gamma} q$, where $\gamma \geq 1$ and $q$ is not a multiple of 3. Since $(0,0) \in V(G)$, Claim 3 (3) implies that the coordinates of each vertex of $G$ are divisible 
by $3^{\gamma}$, so $G$ is isomorphic to a subgraph of $\left(\mathbb{Z} \times \mathbb{Z}\left(N_{1}, \ldots, N_{k}\right), q\right)$, which is 3 -colorable from the previous case.

(3) (a) Suppose $d=2 q$ for some odd $q$. From Claim $2(2)$, $a$ and $b$ have the same parity. Let $E(G)=$ $E_{1} \cup E_{2}$, where $E_{1}=\left\{\{\mathbf{u}, \mathbf{v}\} \in E(G): a_{\mathbf{u}-\mathbf{v}} \equiv 0(\bmod 2)\right\}, E_{2}=E-E_{1}$. Let $G_{i}$ be the subgraph of $G$ such that $E\left(G_{i}\right)=E_{i}, i=1,2$. By considering the directed differences between the rational coordinates of the vertices in $G_{2}$, we conclude that there are no odd cycles in this subgraph, and so $\chi\left(G_{2}\right)=2$. Since $a_{\mathbf{u}-\mathbf{v}} \equiv 0(\bmod 2)$ for each edge $\{\mathbf{u}, \mathbf{v}\}$ in $G_{1}$, by translation and Claim 2 (2) each connected component of $G_{1}$ is isomorphic to a subgraph of $\left(\mathbb{Z} \times \mathbb{Z}\left(N_{1}, \ldots, N_{k}\right), q\right)$, so $\chi\left(G_{1}\right)=2$ by Case 1 . Let $c_{1}$ and $c_{2}$ be the proper 2-colorings of $G_{1}$ and $G_{2}$, respectively. Then $c(\mathbf{v})=\left(c_{1}(\mathbf{v}), c_{2}(\mathbf{v})\right), \mathbf{v} \in V(G)$, is a proper 4-coloring of $G$, so $\chi(G) \leq 4$.

(b) Suppose $N_{i} \not \equiv 7(\bmod 8)$ for $i=1, \ldots, k$ and $d=2^{\gamma} q$, where $\gamma \geq 2$ and $q$ is odd. Claim 2 (4) implies that the coordinates of vertices of $G$ are divisible by $2^{\gamma-1}$, so $G$ is isomorphic to a subgraph of $\left(\mathbb{Z} \times \mathbb{Z}\left(N_{1}, \ldots, N_{k}\right), 2 q\right)$, which is 4 -colorable from the previous case.

(4) First, suppose that $d \not \equiv 0(\bmod 6)$. Then $d \not \equiv 0(\bmod 2)$ or $d \not \equiv 0(\bmod 3)$. In either case, we have a 2 - or 3 -coloring from above. Now, if $d \equiv 6(\bmod 12)$, then $d \equiv 2(\bmod 4)$, in which case we have a 4-coloring. Thus, we may assume that $d \equiv 0(\bmod 12)$, and that $N_{i} \not \equiv 11(\bmod 12)$ for $i=1, \ldots, k$. Define

$$
A=\left\{A_{1}, \ldots, A_{m}\right\}=\left\{N_{i}: N_{i} \not \equiv 3 \quad(\bmod 4)\right\} \text { and } B=\left\{B_{1}, \ldots, B_{m^{\prime}}\right\}=\left\{N_{1}, \ldots, N_{k}\right\} \backslash A .
$$

Note that $B_{i} \not \equiv 2(\bmod 3)$ since $N_{i} \not \equiv 11(\bmod 12)$, and so $B_{i} \equiv 3$ or $7(\bmod 12), i=1, \ldots, m^{\prime}$. For each vertex $\mathbf{v}$, we may uniquely write $\mathbf{v}=\mathbf{v}_{\mathbf{1}}+\mathbf{v}_{\mathbf{2}}$, where $\mathbf{v}_{\mathbf{1}}=\left(a_{0}, a_{1} \sqrt{A_{1}}+\cdots+a_{m} \sqrt{A_{m}}\right)$ and $\mathbf{v}_{\mathbf{2}}=\left(0, b_{1} \sqrt{B_{1}}+\cdots+b_{m^{\prime}} \sqrt{B_{m^{\prime}}}\right)$. Note that from the previous parts, there is a proper 2-coloring $c_{1}$ of $\left(\mathbb{Z} \times \mathbb{Z}\left(A_{1}, \ldots, A_{m}\right), d\right)$ and a proper 3-coloring $c_{2}$ of $\left(\mathbb{Z} \times \mathbb{Z}\left(B_{1}, \ldots, B_{N}\right), d\right)$. We now define $c(\mathbf{v})=\left(c_{1}\left(\mathbf{v}_{\mathbf{1}}\right), c_{2}\left(\mathbf{v}_{\mathbf{2}}\right)\right)$. Clearly, $c$ is a 6-coloring. Now, suppose that $\mathbf{u}=\mathbf{u}_{\mathbf{1}}+\mathbf{u}_{\mathbf{2}}$ is adjacent to $\mathbf{v}$. Then $\mathbf{u}-\mathbf{v}=\left(a, b \sqrt{N_{i}}\right)$ for some $i$. If $N_{i}=A_{j}$ for some $j$, then $c_{1}\left(\mathbf{u}_{\mathbf{1}}\right) \neq c_{1}\left(\mathbf{v}_{\mathbf{1}}\right)$. Otherwise, $N_{i}=B_{j}$ for some $j$ and so $c_{2}\left(\mathbf{u}_{\mathbf{2}}\right) \neq c_{2}\left(\mathbf{v}_{\mathbf{2}}\right)$. In either case, we have $c(\mathbf{u}) \neq c(\mathbf{v})$ and so $c$ is a proper 6 -coloring.

3. On the Chromatic number of $(X, 1)$, where $X$ is a Set of Points Forming a Convex $|X|$-Gon

Let $V$ be a set of $n$ points in convex position in the plane. Let $f(V)$ be the number of pairs of points in $V$ at distance 1. Let $f(n)=\max \left\{f(V):|V|=n, V \subseteq \mathbb{R}^{2}, V\right.$ is in convex position $\}$. It was conjectured that $f(n) \leq 2 n-2$, by Erdős and Moser [8], Erdős and Fishburn [7]. To date, the best upper bound on $f(n)$ is $C n \log n$ for a constant $C$, and is due to Füredi [10] and Braß and Pach [3]. The best lower bound, $f(n) \geq 2 n-7$, is due to Edelsbrunner and Hajnal [6]. As we investigate this problem, we shall interpret most of the results in terms of a graph $G(V)$ with vertex set $V$ and an edge set consisting of the pairs of vertices at distance 1 . Thus 


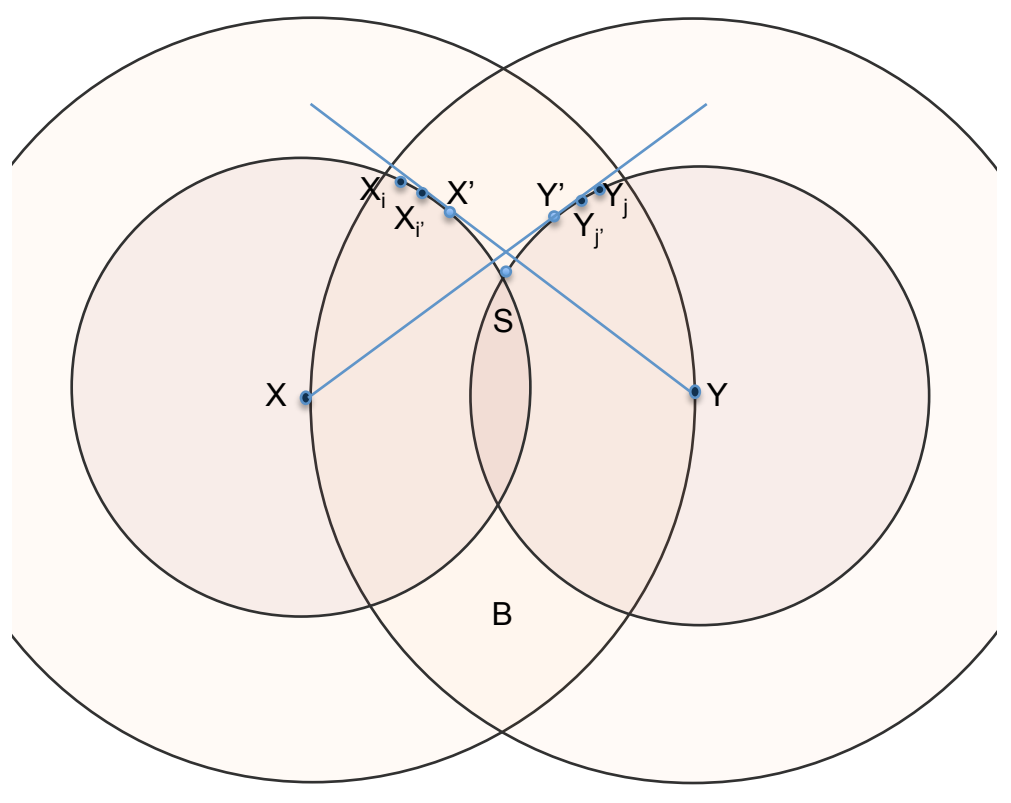

Figure 1. Two points $X, Y$ at a maximum distance and their neighbors.

$f(V)=|E(G(V))|$. Here, we investigate this problem. Let $B_{r}(A)$ be a ball of radius $r$ with center $A$, and let $S_{r}(A)$ be a circle of radius $r$ with center $A$.

Lemma 3.1. Let $V$ be a subset of the plane in convex position with diameter greater than $\sqrt{2}$, then there is a vertex of degree at most 3 in $G(V)$.

Proof. Let $d$ be the diameter of $V, d>\sqrt{2}$. Consider two points $X, Y$ at distance $d$. Assume, without loss of generality, that the line through $X$ and $Y$ is horizontal. Assume that the degree of each vertex in $G(V)$ is at least 4 .

Idea of the proof:

Consider 4 neighbors of $X$ and 4 neighbors of $Y$. We shall first observe that at most two of the neighbors of $X$ could be in the "middle" arc of the unit circle centered at $X$, otherwise $V$ is not in a convex position. Next, we observe that if a neighbor of $X$ is in the "upper" arc, and a neighbor of $Y$ is in the "lower" arc of the corresponding circles, then the distance between these neighbors is greater than $d$, contradicting the diameter condition. Thus, without loss of generality there must be two neighbors of $X$ and two neighbors of $Y$ in the "upper" part of the corresponding circles. See Figure 1. These four points, together with $X$ and $Y$ are not in convex position, resulting in a final contradiction. 
For a formal argument, we introduce some notation. Let, the sets of neighbors, $N(X)=\left\{X_{1}, \ldots, X_{p}\right\} \subseteq$ $S_{1}(X)$ and $N(Y)=\left\{Y_{1}, \ldots, Y_{q}\right\} \subseteq S_{1}(Y)$ with $p, q \geq 4$. Since $\operatorname{diam}(V)=d$, we have that $V \subseteq B=$ $B_{d}(X) \cap B_{d}(Y)$. Let us denote the half-plane above line $X Y$ as $P_{\text {up }}$, and the half-plane below $X Y$ as $P_{d o w n}$. Let $X^{\prime}, X^{\prime \prime}, Y^{\prime}, Y^{\prime \prime}$ be "tangent" points, $Y^{\prime} \in S_{1}(Y) \cap P_{u p}, X^{\prime} \in S_{1}(X) \cap P_{u p}, Y^{\prime \prime} \in S_{1}(Y) \cap P_{\text {down }}, X^{\prime \prime} \in$ $S_{1}(X) \cap P_{\text {down }}$ such that $X Y^{\prime}$ and $X Y^{\prime \prime}$ are tangent to $S_{1}(Y), Y X^{\prime}$ and $Y X^{\prime \prime}$ are tangent to $S_{1}(X)$. These tangent points $X^{\prime}$ and $X^{\prime \prime}$ split $S_{1}(X) \cap B$ into the three arcs mentioned above, and so do $Y^{\prime}, Y^{\prime \prime}$ with $S_{1}(Y) \cap B$.

Let $S_{1}(X) \cap B=S_{u}(X) \cup S_{m}(X) \cup S_{l}(X)$, let $S_{1}(Y) \cap B=S_{u}(Y) \cup S_{m}(Y) \cup S_{l}(Y)$, where $u, m, l$ stand for upper, middle, lower parts, and $S_{m}(X)$ is the $X^{\prime}-X^{\prime \prime}$ arc of $S_{1}(X), S_{u}(X)$ is the arc of $S_{1}(X) \cap B$ above $S_{m}(X)$, and $S_{l}(X)$ is the arc of $S_{1}(X) \cap B$ below $S_{m}(X)$. We define $S_{u}(Y), S_{m}(Y), S_{l}(Y)$ similarly. Note that $X Y^{\prime} Y X^{\prime \prime}$ and $X X^{\prime} Y Y^{\prime \prime}$ are rectangles with diagonals of length $d$ and one of the side lengths 1.

Claim: The distance between $S_{l}(X)$ and $S_{u}(Y)$ is greater than $d$.

To prove this claim, consider two lines $\ell_{x}, \ell_{y}$ - one passing through $X^{\prime \prime}$, another through $Y^{\prime}$ such that they are perpendicular to $X^{\prime \prime} Y^{\prime}$. The distance between these lines is $d$. We see that for $d>1$, the slopes of $\ell_{x}$ and $\ell_{y}$ are negative. Thus $S_{l}(X)$ and $S_{u}(Y)$ are outside of the plane region between $\ell_{x}$ and $\ell_{y}$, and so the distance between $S_{l}(X)$ and $S_{u}(Y)$ is greater than the distance between $\ell_{x}$ and $\ell_{y}$, namely $d$.

Observe that there is at most one point of $V$ in each of $S_{m}(Y) \cap P_{u p}, S_{m}(Y) \cap P_{\text {down }}, S_{m}(X) \cap P_{\text {up }}$, and $S_{m}(X) \cap P_{\text {down }}$ because of convexity. Since $|N(X)|,|N(Y)| \geq 4$, and because of the claim, there are, without loss of generality, $X_{i}, X_{i^{\prime}} \in S_{u}(X)$, and $Y_{j}, Y_{j^{\prime}} \in S_{u}(Y)$. Note that the slope of $X_{i} X_{i^{\prime}}$ line is negative and the slope of $Y_{j} Y_{j^{\prime}}$ is positive. This contradicts the convexity of $V$ since, for $d>\sqrt{2}, S_{u}(X)$ is completely to the left of $S_{u}(Y)$.

Corollary 3.2. Let $V$ be a subset of a plane in convex position, then $\chi(G(V)) \leq 4$.

Proof. If $V$ has diameter at most $\sqrt{2}$, it could be embedded into a square with side length $\sqrt{2}$. This square could be four-colored by splitting in into four squares $s_{1}, s_{2}, s_{3}, s_{4}$ of equal sizes and assigning color $i$ to each point of square $s_{i}$.

4. On the Chromatic number of $(X, 1)$, where $X \subset \mathbb{R}^{2}$ For $X$ An Infinite Strip and for $X$ a union OF LINES

Pritikin [15] produced a 7-coloring of the plane such that one color class, say of color 0, is small. Using this coloring, it was shown that any 6197 points can be simultaneously translated so that none of the points are assigned color 0 . In fact, a stronger statement was proven.

A good 7-coloring of the plane is a coloring using colors $0,1, \ldots, 6$, such that if there are two vertices at distance 1 having the same color, this color is 0 . 


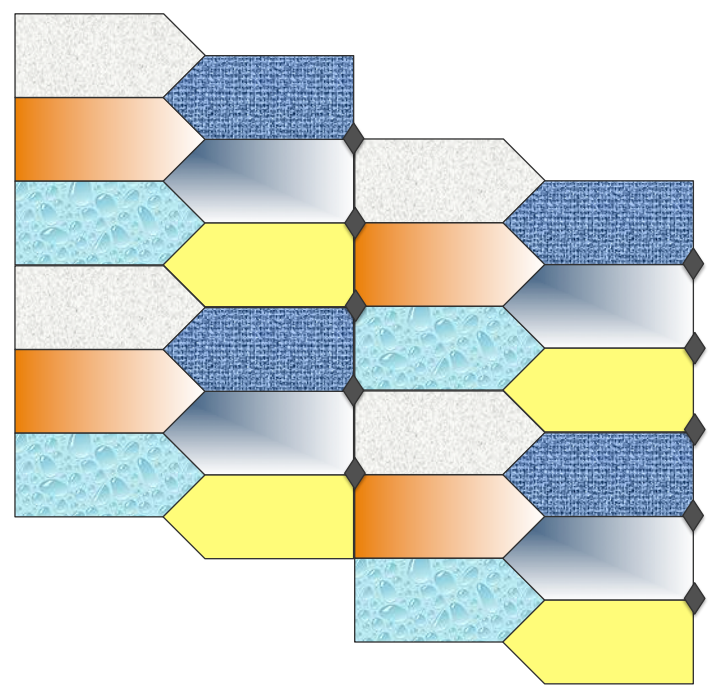

Figure 2. A tiling of the plane with tiles of 7 different colors, the tiles in each column use 3 different colors, every other column has the same coloring (shifted)

Theorem 4.1 (Pritikin [15]). Let c be a good 7-coloring of the plane which is periodic, i.e., there are $\alpha, \beta, \gamma>0$ such that for all $(x, y) \in \mathbb{R}^{2}, c(x, y)=c(x, y+\beta)=c(x+\alpha, y+\gamma)$. Let $S=\{(x, y): 0 \leq x<\alpha, 0 \leq y<$ $\beta, c(x, y)=0\}$ be a Riemann integrable set of area $A>0$. If $|X|<\alpha \beta / A$, then $\chi(X, 1) \leq 6$.

The main idea of Pritikin's construction is to consider a pentagonal tiling of the plane (of type 5, according to Wolfram Alpha classification of 14 known pentagonal tilings), color it periodically with colors $1, \ldots, 6$, and then recolor small diamond-shaped regions with the color 0. See Figure 2 for an illustration.

In [1] and [13], the chromatic numbers of regions of the plane, such as circular or rectangular regions, are investigated. Several interesting precise results are proven in those papers with regards to 3-colorable regions. In particular, Bauslaugh [1] proves that an infinite strip in the plane is 3-colorable if and only if it has width at most $\sqrt{3} / 2$. Finding such a sharp result for 4 -colorable strips seems to be difficult, and investigating 5 and 6 -colorable strips is probably as difficult as the original question. However, we can provide the lower bounds on the width of an $i$-colorable strip in the plane, for $i=4,5,6$ using constructions. Let $\omega(i)$ be the largest width of an $i$-colorable strip in the plane.

\section{Theorem 4.2.}

$$
\omega(i) \geq \begin{cases}\sqrt{3} / 2 \geq 0.866, & i=3, \\ 2 \sqrt{2} / 3 \geq 0.94, & i=4, \\ \sqrt{15} / 4 \geq 0.968, & i=5, \\ \sqrt{15} / 2+\sqrt{3} \geq 3.668, & i=6 .\end{cases}
$$

Proof. The fact that $\omega(3) \geq \sqrt{3} / 2$ is a theorem proved by Bauslaugh [1]. To see that $\omega=\omega(4) \geq \sqrt{8 / 9}$, as mentioned in [1], construct the colorings of an infinite strip of width $\omega$ built of monochromatic $\omega \times 1 / 3$ rectangles 
of colors $1,2,3,4,1,2,3,4, \ldots$, see Figure 3 . To see that $\omega=\omega(5) \geq \sqrt{15 / 16}$, build a similar construction with monochromatic $\omega \times 1 / 4$ rectangles. To observe that $\omega(6) \geq \sqrt{15} / 2+\sqrt{3}$, we consider a coloring, similar to one used by Pritikin, see Figure 4 . The symmetric tiles have width $1 / 2$, with the rounded boundary being a circular arc of radius 1 centered at the opposite corner of the tile. The tiles used in the top and bottom rows are determined by the distance 1 between the point of intersection of circular arcs and the opposite corner of a tile.

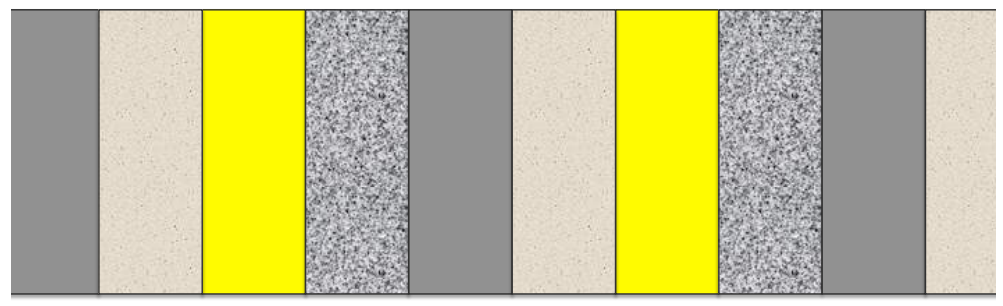

FigURE 3. A coloring of an infinite strip using 4 colors

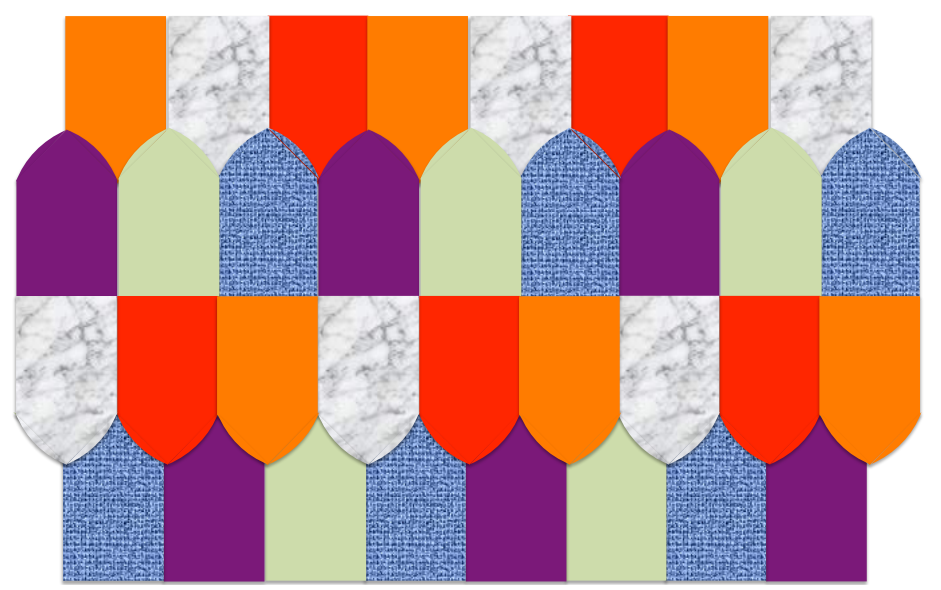

Figure 4. A coloring of an infinite strip using 6 colors

Theorem 4.3. Let $X$ be the union of two parallel lines on the plane. If the distance $\ell$ between two lines satisfies one of 
a) $\ell \geq 1$,

b) $\ell<1$ and $\sqrt{1-\ell^{2}}$ is irrational, or

c) $\ell<1$ and $\sqrt{1-\ell^{2}}$ is rational with an odd denominator in its lowest term,

then $\chi(X, 1)=2$. Otherwise, $\chi(X, 1)=3$.

Proof. Assume that $X$ is the union of vertical lines $L_{1}$ and $L_{2}$ at distance $\ell$. If $\ell>1$, then we can color $L_{1}$ and $L_{2}$ in two colors independently. If $\ell=1$, then, for each vertex in $L_{1}$, there is exactly one vertex in $L_{2}$ adjacent to it, namely, a vertex with the same $y$-coordinate. Let $c$ be a proper coloring of $\left(L_{1}, 1\right)$ with colors 0 and 1. For $x \in L_{2}$, let $c(x)=1-c\left(x^{\prime}\right)$, where $x^{\prime} \in L_{1}$ and $x^{\prime}$ has the same $y$-coordinate as $x$. This gives a proper 2 -coloring of $(X, 1)$ when $\ell=1$. So, assume for the rest of the proof that $\ell<1$. Consider a finite subset $X^{\prime}$ of $X$ and a point in $X^{\prime}$ with the largest $y$-coordinate. It is adjacent to at most 2 other vertices in $\left(X^{\prime}, 1\right)$. By induction on the size of $X^{\prime}$, it follows that $\chi\left(X^{\prime}, 1\right) \leq 3$. Theorem 1.1 then gives that $\chi(X, 1) \leq 3$.

We shall now prove that $\chi(X, 1)=2$ when $\ell$ satisfies conditions b) or $\mathrm{c}$ ) of the theorem. Assume that $(0,0) \in L_{1}$. Let $L$ be the connected component of $(X, 1)$ containing $(0,0)$. Then the $y$-coordinate, $u_{y}$, of any point $u$ in $V(L)$ can be expressed in the form $u_{y}=a+b \sqrt{1-\ell^{2}}$, where $a, b \in \mathbb{Z}$, and $b$ is even if $u \in L_{1}, b$ is odd if $u \in L_{2}$. This fact can be easily verified by induction on $|V(L)|$. We shall refer to such a representation $u_{y}$ of $u$ as a good representation.

Case 1. Either $\sqrt{1-\ell^{2}}$ is irrational or $\sqrt{1-\ell^{2}}=p / q, p, q \in \mathbb{Z}, q$-odd.

Claim. For any two good representations of a point in $L, a+b \sqrt{1-\ell^{2}}=a^{\prime}+b^{\prime} \sqrt{1-\ell^{2}}$, we have $a \equiv a^{\prime}$ $(\bmod 2)$ and $b \equiv b^{\prime}(\bmod 2)$.

Since the representations are good, we have immediately that $b \equiv b^{\prime}(\bmod 2)$. Let $\sqrt{1-\ell^{2}}$ be irrational. Since $a-a^{\prime}+\left(b-b^{\prime}\right) \sqrt{1-\ell^{2}}=0$, the linear independence of irrational numbers over rational numbers gives that $b=b^{\prime}$ and thus $a=a^{\prime}$. Let $\sqrt{1-\ell^{2}}=\frac{p}{q}$, where $p, q \in \mathbb{Z}$ and $q$ is odd. So, $a-a^{\prime}+\left(b-b^{\prime}\right) \sqrt{1-\ell^{2}}=0$, and $q\left(a-a^{\prime}\right)+\left(b-b^{\prime}\right) p=0$. Since $q$ is odd and $b-b^{\prime}$ is even, $a-a^{\prime}$ must be even. This verifies the claim.

For a vertex $u$ with good representation $a+b \sqrt{1-\ell^{2}}$, let the color of $u, c(u)=a+b(\bmod 2)$. From the Claim, the coloring is well-defined. Since any points on the same line $L_{i}$ have the same parity for a coefficient in front of $\sqrt{1-\ell^{2}}$, it gives a proper coloring of $L_{i}, i=1,2$. Assume that $u \in L_{1}, v \in L_{2}$ are two adjacent vertices of the same color. Let $u_{y}$ and $v_{y}$ be good representations of $u$ and $v: u_{y}=a+b \sqrt{1-\ell^{2}}, v_{y}=a^{\prime}+b^{\prime} \sqrt{1-\ell^{2}}$. Note that since $b$ and $b^{\prime}$ have different parities and $c(u)=c(v), a$ and $a^{\prime}$ must have different parities. Since $\|u-v\|^{2}=1=\ell^{2}+\left(\left(a-a^{\prime}\right)+\left(b-b^{\prime}\right) \sqrt{1-\ell^{2}}\right)^{2}$, we have that $\left(a-a^{\prime}\right)+\left(b-b^{\prime} \pm 1\right) \sqrt{1-\ell^{2}}=0$.

If $\sqrt{1-\ell^{2}}$ is irrational and $a \neq a^{\prime}$, we have a contradiction. If $\sqrt{1-\ell^{2}}=\frac{p}{q}, p, q \in \mathbb{Z}$ and $q$ is odd, then $\left( \pm 1-b+b^{\prime}\right) \frac{p}{q}=\left(a-a^{\prime}\right)$, so $\left( \pm 1-b+b^{\prime}\right) p=q\left(a-a^{\prime}\right)$. Here, the left-hand side is even and the right-hand side is odd, a contradiction. 
Case 2. $\sqrt{1-\ell^{2}}=\frac{p}{2 q}, p, q \in \mathbb{Z}, \operatorname{gcd}(p, 2 q)=1$.

Observe that $L$ contains an odd cycle with vertices $(0,0),\left(\ell, \sqrt{1-\ell^{2}}\right),\left(0,2 \sqrt{1-\ell^{2}}\right), \ldots,\left(\ell,(2 q-1) \sqrt{1-\ell^{2}}\right)$, $\left(0,2 q \sqrt{1-\ell^{2}}\right)=(0, p),(0, p-1), \ldots,(0,1),(0,0)$, so $\chi(X, 1) \geq 3$ in this case.

Acknowledgements The authors would like to thank the Department of Mathematics at Iowa State University for supporting this graduate research project and Felix Lazebnik for bringing the manuscript of Moorehouse to their attention.

\section{REFERENCES}

[1] B. L. Bauslaugh. Tearing a strip off the plane. J. Graph Theory, 29(1):17-33, 1998.

[2] I. Boreico. My favorite problem: linear independence of radicals. Harv. Coll. Math. Rev., 2:87-92, 2008.

[3] P. Braß and J. Pach. The maximum number of times the same distance can occur among the vertices of a convex $n$-gon is $O(n \log n)$. J. Combin. Theory Ser. A, 94(1):178-179, 2001.

[4] K. B. Chilakamarri. The unit-distance graph problem: a brief survey and some new results. Bull. Inst. Combin. Appl., 8:39-60, 1993.

[5] N. G. de Bruijn and P. Erdős. A colour problem for infinite graphs and a problem in the theory of relations. Nederl. Akad. Wetensch. Proc. Ser. A. 54 = Indagationes Math., 13:369-373, 1951.

[6] H. Edelsbrunner and P. Hajnal. A lower bound on the number of unit distances between the vertices of a convex polygon. J. Combin. Theory Ser. A, 56(2):312-316, 1991.

[7] P. Erdős and P. C. Fishburn. Multiplicities of interpoint distances in finite planar sets. Discrete Appl. Math., 60(1-3):141-147, 1995. ARIDAM VI and VII (New Brunswick, NJ, 1991/1992).

[8] P. Erdős and L. Moser. Problem 11. Canadian Math. Bull., 2:43, 1959.

[9] K. G. Fischer. Additive K-colorable extensions of the rational plane. Discrete Math., 82(2):181-195, 1990.

[10] Z. Füredi. The maximum number of unit distances in a convex n-gon. J. Combin. Theory Ser. A, 55(2):316-320, 1990.

[11] P. D. Johnson, Jr. Two-colorings of real quadratic extensions of $Q^{2}$ that forbid many distances. Congr. Numer., 60:51-58, 1987. Eighteenth Southeastern International Conference on Combinatorics, Graph Theory, and Computing (Boca Raton, Fla., 1987).

[12] M. Klazar. A question on linear independence of square roots. Unpublished.

[13] C. P. Kruskal. The chromatic number of the plane: the bounded case. J. Comput. System Sci., 74(4):598-627, 2008.

[14] G. E. Moorehouse. On the chromatic numbers of planes. Preprint.

[15] D. Pritikin. All unit-distance graphs of order 6197 are 6-colorable. J. Combin. Theory Ser. B, 73(2):159-163, 1998.

[16] A. Soifer. The mathematical coloring book. Springer, New York, 2009. Mathematics of coloring and the colorful life of its creators, With forewords by Branko Grünbaum, Peter D. Johnson, Jr. and Cecil Rousseau.

[17] D. R. Woodall. Distances realized by sets covering the plane. J. Combinatorial Theory Ser. A, 14:187-200, 1973.

\section{Appendix - Proofs of Claims from Section 2}

Proof of Claim 1. We have that $\mathbf{w}=\mathbf{u}-\mathbf{v}$ is a vector of length $d, d^{2}=a_{w}^{2}+\left(\sum_{i=1}^{k} b_{w i} \sqrt{N_{i}}\right)^{2}$. Thus $\sum_{i=1}^{k} b_{w i} \sqrt{N_{i}}$ is the square root of an integer, $d^{2}-a_{w}^{2}$. We know that any set consisting of distinct square free integers is linearly independent over the field $\mathbb{Q}$ (this is from a simple fact of quadratic field extensions, or see, for example, [2], [12]). Thus all but at most one expression $b_{w i}$ is zero. 


\section{Proof of Claim 2.}

(1) We have that $a+b N \equiv a^{2}+b^{2} N=d^{2} \equiv d \equiv 1(\bmod 2)$.

(2) We have that $a^{2}+b^{2} N \equiv 0(\bmod 4)$. Since $N$ is square free, $a$ is even if and only if $b$ is even.

(3) Suppose $a$ and $b$ are odd. Then, from $a^{2}+b^{2} N=d^{2}$, we have $1+N \equiv 0(\bmod 4)$, which is impossible if $N \not \equiv 3(\bmod 4)$. Hence from $(2), a$ and $b$ are even. By induction on $\gamma \geq 1$, we are done.

(4) Similar to (3), but consider modulo 8 and induction on $\gamma \geq 2$.

\section{Proof of Claim 3}

(1) Suppose $d \not \equiv 0(\bmod 3)$. Hence $d^{2} \equiv 1(\bmod 3)$. So $a^{2}+b^{2} N \equiv 1(\bmod 3)$. If $N \equiv 0(\bmod 3)$, then $a^{2} \equiv 1(\bmod 3)$, i.e. $a \not \equiv 0(\bmod 3)$. Thus $a+b N \not \equiv 0(\bmod 3)$. If $N \equiv 1(\bmod 3)$, then $a^{2}+b^{2} \equiv 1(\bmod 3)$, i.e. $\left(a^{2} \equiv 1(\bmod 3)\right.$ and $\left.b^{2} \equiv 0(\bmod 3)\right)$ or $\left(a^{2} \equiv 0(\bmod 3)\right.$ and $\left.b^{2} \equiv 1(\bmod 3)\right)$. In either case, we have $a+b N \not \equiv 0(\bmod 3)$. If $N \equiv 2(\bmod 3)$, then $a^{2}+2 b^{2} \equiv 1(\bmod 3)$, i.e. $a^{2} \equiv 1(\bmod 3)$ and $b^{2} \equiv 0(\bmod 3)$. So $a \not \equiv 0(\bmod 3)$ and $b \equiv 0(\bmod 3)$. Therefore we have $a+b N \not \equiv 0(\bmod 3)$.

(2) Suppose $a^{2} \not \equiv b^{2}(\bmod 3)$. So one of $a$ and $b$ is a multiple of 3 . Since $N$ is square free, the other should be a multiple of 3 , i.e. $a^{2} \equiv b^{2} \equiv 0(\bmod 3)$, a contradiction.

(3) Suppose $d \equiv 0(\bmod 3)$ and $N \not \equiv 2(\bmod 3)$. Hence $a^{2}+b^{2} N \equiv 0(\bmod 3)$. If $a^{2} \equiv b^{2} \equiv 1(\bmod 3)$, then we have $1+N \equiv 0(\bmod 3)$, which is impossible since $N \not \equiv 2(\bmod 3)$. From $(2), a^{2} \equiv b^{2} \equiv 0(\bmod 3)$, i.e. $a \equiv b \equiv 0(\bmod 3)$. Then by induction on $\gamma \geq 1$, we are done.

Department of Mathematics, Iowa State University, Ames, IA 50011

E-mail address: axenovic@iastate.edu, jchoi@iastate.edu, lastrina@iastate.edu, tmckay16@iastate.edu, smithj@iastate.edu, bstanton@iastate.edu 